\title{
Karst et paléo-écoulements dans le Pays-Haut (Lorraine) : l'exemple du bassin versant de la vallée d'Anderny-Malavillers
}

Karst and palaeodrainage in the Pays-Haut, Lorraine: the example of the valley of Anderny-Malavillers drainage basin

Karst und Paläoabfluss im Pays-Haut. Beispiel des Einzugsgebiets des AndernyMalavillers-Tales

Patrice Gamez, Dominique Harmand et Florence Caramelle

\section{OpenEdition}

Journals

Édition électronique

URL : http://journals.openedition.org/rge/4221

DOI : $10.4000 /$ rge. 4221

ISSN : $2108-6478$

Éditeur

Association des géographes de l'Est

Édition imprimée

Date de publication : 1 janvier 2000

ISSN : 0035-3213

\section{Référence électronique}

Patrice Gamez, Dominique Harmand et Florence Caramelle, « Karst et paléo-écoulements dans le Pays-Haut (Lorraine) : l'exemple du bassin versant de la vallée d'Anderny-Malavillers », Revue Géographique de l'Est [En ligne], vol. 40 / 1-2 | 2000, mis en ligne le 06 août 2013, consulté le 10 décembre 2020. URL : http://journals.openedition.org/rge/4221 ; DOI : https://doi.org/10.4000/rge. 4221

Ce document a été généré automatiquement le 10 décembre 2020.

Tous droits réservés 


\section{Karst et paléo-écoulements dans le Pays-Haut (Lorraine) : l'exemple du bassin versant de la vallée d'Anderny-Malavillers}

Karst and palaeodrainage in the Pays-Haut, Lorraine: the example of the valley

of Anderny-Malavillers drainage basin

Karst und Paläoabfluss im Pays-Haut. Beispiel des Einzugsgebiets des Anderny-

Malavillers-Tales

Patrice Gamez, Dominique Harmand et Florence Caramelle

1 Le centre du Pays-Haut se situe dans la Lorraine septentrionale, sur le revers de la côte de Moselle, lequel coïncide largement avec le plateau du Bajocien. (fig. 1). Cette partie de l'ancien bassin ferrifère de Briey est, en premier lieu, affectée par la présence de nombreux phénomènes karstiques, et en second lieu, traversée du nord-ouest au sudest par une vallée sèche: la vallée d'Anderny-Malavillers qui recoupe perpendiculairement la limite des bassins versants de la Meuse et du Rhin. 
Fig. 1 : Cadre morphostructural du Pays-Haut.

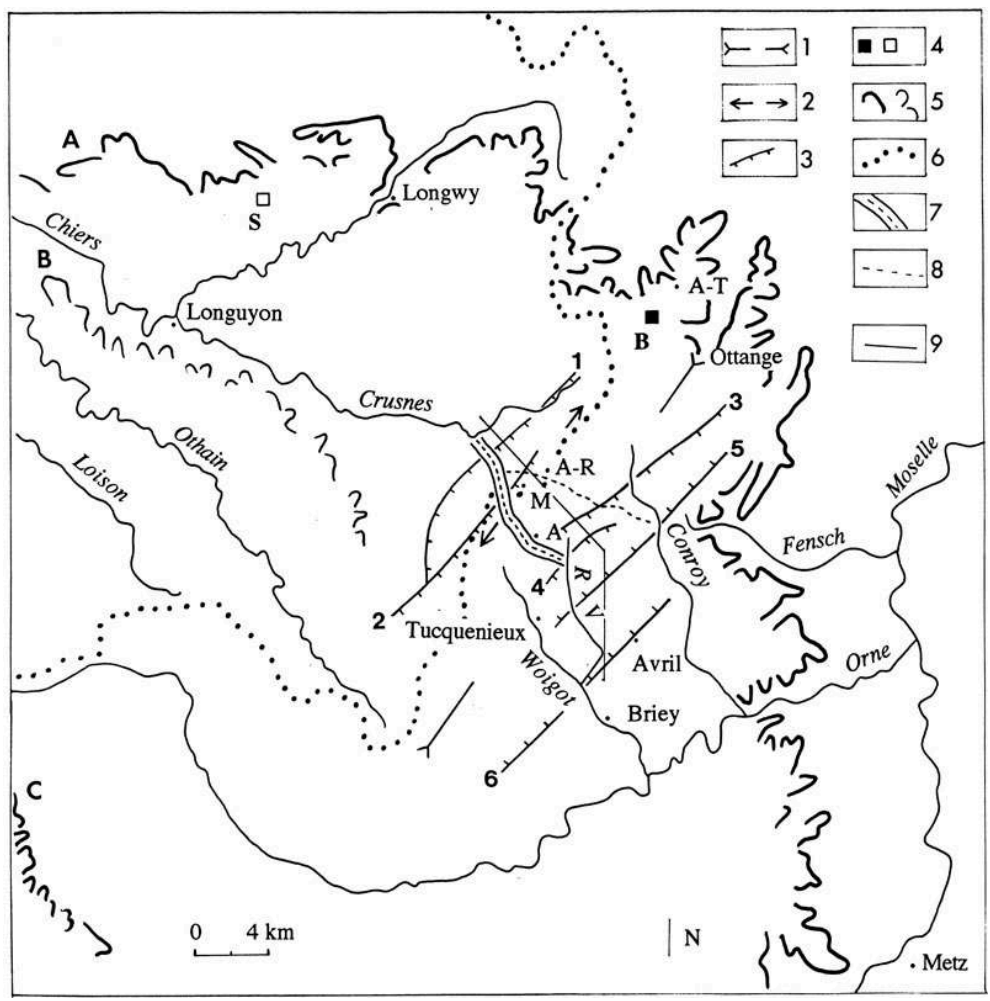

1 : ondulation synclinale d'Ottange-Tucquenieux, 2 : ondulation anticlinale d'Audun-le-Roman, 3 : failles du fossé de Tucquenieux (1.1: de Crusnes, 1.2 : de Mont-Bonvillers, 1.3 : de Sancy, 1.4 : de Brabant, 1.5 : de Bettainvillers, $1.6: d^{\prime}$ Avril), 4 : paléokarsts tertiaires à remplissages ferrugineux (B: Borne de Fer, $S$ : Saint-Pancré), 5 : côtes (A : du Bajocien, B : de la Dalle d'Etain, Bathonien, C : de l'Oxfordien moyen), 6 : limite des bassins versants Rhin/Meuse, 7 : vallée sèche d'Anderny-Malavillers, 8 : vallée sèche d'Audun-le-Roman, 9 : tracé de la figure 2 ; A : Anderny, A-R : Audun-le-Roman, A-T : Audun-le-Tiche, M : Malavillers, RV : Ruisseau de la Vallée.

2 Cette étude se propose de définir la nature et l'évolution d'un type de karst couvert et de retracer les étapes de l'incision du réseau hydrographique du plateau de revers karstifié de la côte de Moselle.

\section{Le cadre morphostructural}

\section{A. La forte commande structurale du karst}

3 Il apparaît de prime abord que la distribution et la nature des phénomènes karstiques sont fondamentalement liées aux particularités de la structure régionale.

\section{La commande tectonique}

4 À l'échelle régionale, le secteur étudié se situe dans le fond de l'ondulation synclinale de Luxembourg, localement dénommée "synclinal d'Ottange-Tucquenieux » dans le secteur de la vallée d'Anderny-Malavillers (fig. 1).

5 A l'échelle locale, cette structure est affectée d'une série de failles d'orientation $\mathrm{N} 30$ à N 60 formant un graben entre les failles d'Audun-le-Tiche au NW et d'Avril au SE : la vallée d'Anderny recoupe les failles du flanc NW de ce fossé. Le jeu subsident de 
l'ondulation synclinale de Luxembourg est attesté du Keuper supérieur au CallovoOxfordien, expliquant ainsi le sur-épaississement des séries stratigraphiques, et en particulier de la formation ferrifère aalénienne du bassin de Briey (Le Roux, 1980). Ce dispositif d'orientation varisque a enregistré les effets d'un régime de décrochement senestre $\mathrm{N} 50-\mathrm{N} 65$ et s'accompagne d'un système de fracturation de deuxième ordre développé : alignement N 50 de fentes en échelon, diaclases N 150-N 170. Cette phase tectonique cassante est imputée à la compression NNE "pyrénéenne ", d'âge éocène. Une phase ultérieure de compression SE (miocène) n'aurait fait que remobiliser les systèmes de fractures subméridiens (Steiner, 1980).

6 Le dispositif tectonique n'a guère eu d'influence sur le tracé du réseau hydrographique qui recoupe plus ou moins obliquement les différentes failles. Mais ce dispositif tectonique a eu une influence marquée sur les écoulements souterrains, en favorisant la création de drains karstiques à écoulements nord-sud (Joly, 1927).

\section{La commande lithologique}

$7 \mathrm{Au}$ Mésozoïque, structures souples et failles ont déterminé des variations rapides et fréquentes de faciès lithologiques (LE ROUX, 1983). Une coupe-type (fig. 2) voit se succéder au-dessus de la formation ferrifère et des Marnes micacées qui la scellent (Bresson, 1960 ; Le Roux, 1983) :

Fig. 2 : Coupe géologique nord-ouest - sud-est à travers le fossé de Tucquenieux

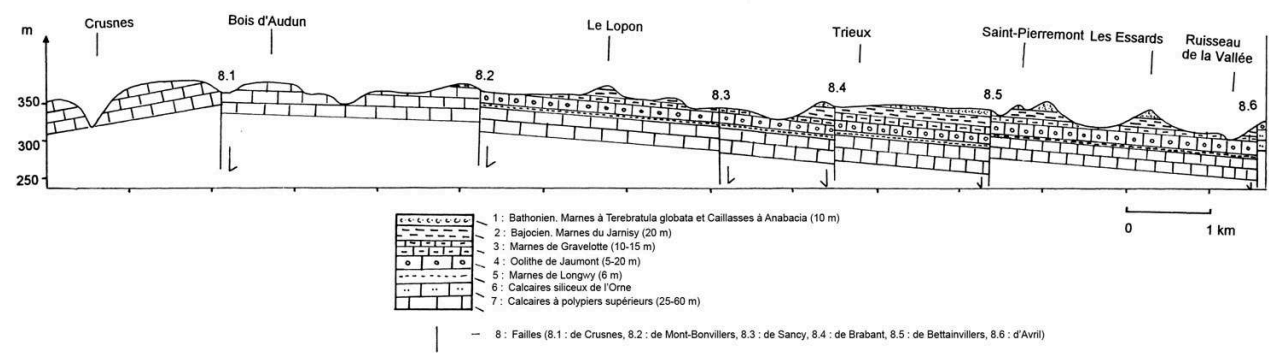

D'après Le Roux, 1983 ; Caramelle, 1998

8 - un premier ensemble essentiellement calcaire couronné par l'Oolithe de Jaumont, qui forme le soubassement de la vallée d'Anderny. Le faciès lenticulaire des Calcaires siliceux (calcaires gréseux à minces interbancs de marnes gréseuses) et les calcaires à polypiers n'affecteraient le secteur étudié qu'anecdotiquement ;

9 - un deuxième ensemble marno-calcaire correspondant au sommet du Bajocien et au Bathonien inférieur. Le déblaiement imparfait du toit de l'Oolithe de Jaumont confère au secteur de la vallée d'Anderny l'allure d'un plateau structural surmonté de modestes buttes-témoins et avant-buttes de la côte mineure des Caillasses à Anabacia. Les buttestémoins des Caillasses à Anabacia sont largement conservées en inversion de relief dans le fossé de Tucquenieux, notamment de part et d'autre de la vallée d'Anderny, entre Trieux et Tucquenieux (fig. 3, 4). 
Fig. 3 : Les phénomènes karstiques et leur cadre morphostructural

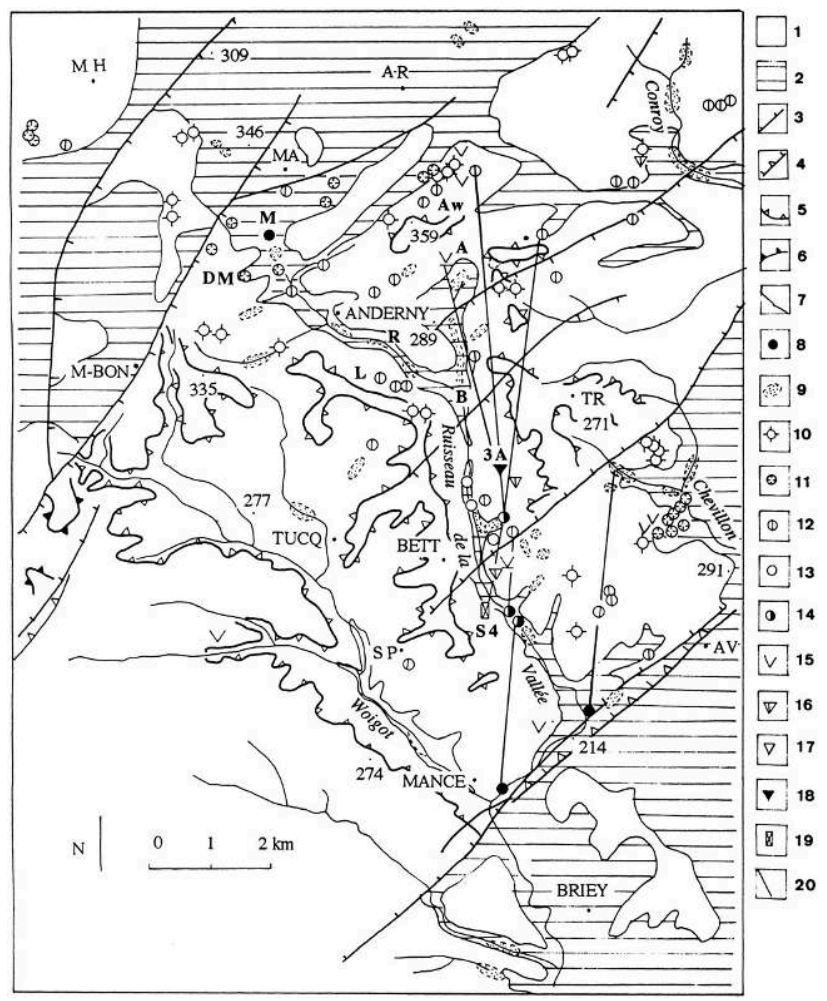

1 : marno-calcaires du Bajocien supérieur et du Bathonien, 2 : calcaires bajociens, 3 : failles, 4 escarpement de ligne de faille, 5 : microcôte des caillasses à Anabacia, $6:$ microcôte des caillasses à Rhynchonelles, 7 : cours d'eau permanents ou temporaires, 8 : source (M : Maufontaine), 9 : perte diffuse (B : Brabant, $R$ : Rouau), 10 : mardelle, 11 : doline (D : Dame Marion), $12:$ doline-perte (Aw : les Awis, L : Linière), 13 : doline-émergence, 14 : doline-inversac, 15 : gouffre (A : gouffre Armand), 16 : gouffre-perte, $17:$ gouffre-regard, 18 : gouffre-émergence, $19:$ grotte-inversac, $20:$ traçage hors contexte minier ; A-R : Audun-le-Roman, AV : Avril, BETT : Bettainvillers, MA : Malavillers, M-H : Mercyle-Haut, M-Bon : Mont-Bonvillers, SA : Sancy, SP : Saint-Pierremont-Cités, TR : Trieux, Tucq : Tucquenieux. 
Fig. 4 : Carte géomorphologique de la vallée d'Anderny

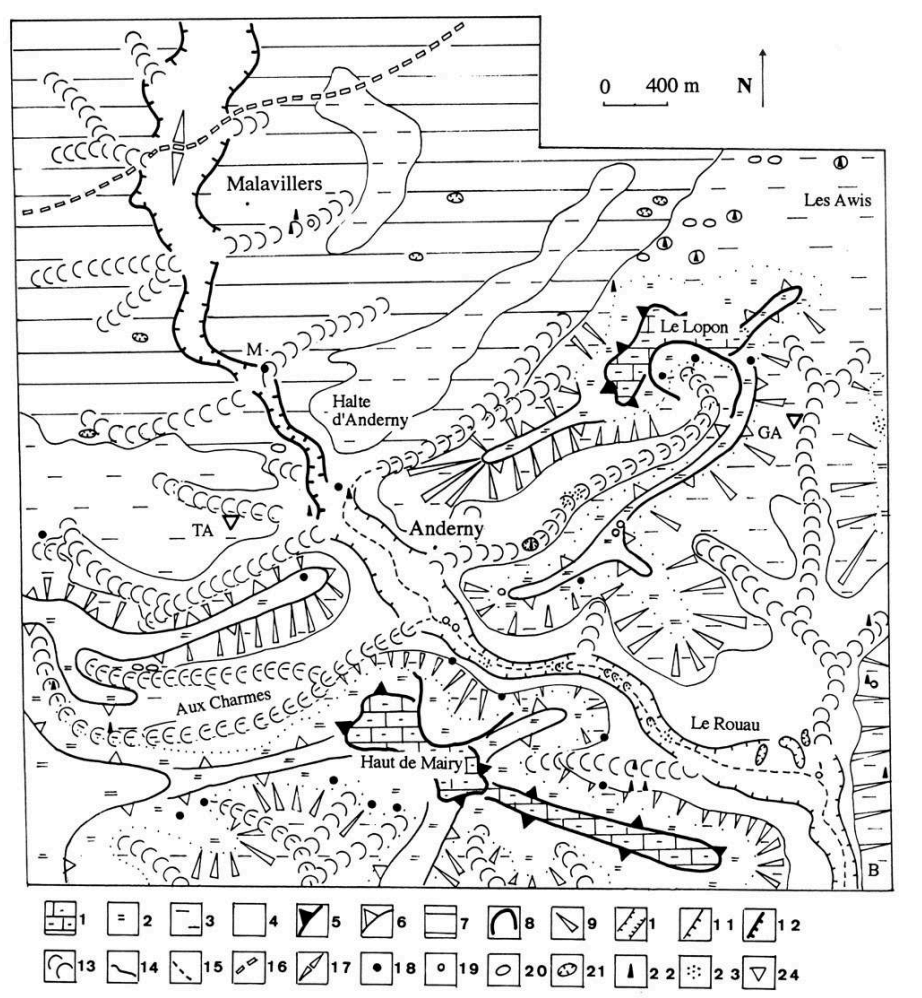

1 : Caillasse à Anabacia, 2 : Marnes du Jarnisy, 3 : Marnes de Gravelotte, 4 : Oolithe de Jaumont, 5 : Buttes-témoins de la Caillasse à Anabacia, 6 : Avant-buttes des Marnes du Jarnisy, 7 : Surface structurale de l'Oolithe de Jaumont, 8 : Rondvaux, 9 : Glacis dans les Marnes de Gravelotte, 10 : Fond de la vallée d'Anderny-Malavillers, 11 : Versants de moins de $15 \mathrm{~m}$ de commandement, 12 : Versants de plus de $15 \mathrm{~m}$ de commandement, 13 : Vallons secs ou à écoulement temporaire, 14 : Écoulement pérenne, 15 : Écoulement temporaire, 16 : Limite des bassins versants de la Meuse (au nord) et de la Moselle (au sud), 17 : Col de Malavillers, 18 : Émergence, 19 : Rejets d'effluents, 20 : Mardelle, 21 : Doline, 22 : Perte, 23 : Perte diffuse, 24 : Gouffre, B : Cités de Brabant, GA : Gouffre Armand, M : Source de Maufontaine, TA : Trou d'Anderny.

Le massif karstifié des calcaires de Jaumont $(10-25 \mathrm{~m})$ est donc recouvert par une couverture marno-calcaire semi-perméable nettement plus épaisse $(65 \mathrm{~m}$, Caillasses à Rhynchonelles comprises) lorsqu'elle est complète.

\section{B. La vallée sèche d'Anderny-Malavillers}

Encaissée d'une quarantaine de mètres dans les calcaires bajociens, la vallée d'AndernyMalavillers, présente en fait, deux vallées de part et d'autre du « col» de Malavillers, col situé à 338 m d'altitude, à la limite des bassins versants de la Meuse et du Rhin.

$\mathrm{Au}$ NW, la vallée de Malavillers est une vallée sèche tributaire du bassin versant de la Meuse, et se situe dans le prolongement de la vallée moyenne de la Crusnes, affluent de la Chiers. Au SE, la vallée d'Anderny sensu lato est une vallée temporairement drainée appartenant au bassin de la Moselle, dont les eaux rejoignent le Ruisseau de la Vallée, sous-affluent de l'Orne (fig. 1). Or, le tracé des vallées sèches de la région d'Audun-leRoman met clairement en évidence une extension ancienne du bassin de la Meuse vers le SE, sur le revers de la côte de Moselle. D'une part, les vallées sèches d'AndernyMalavillers et d'Audun-le-Roman convergent au NW de cette der-nière localité, en 
direction de la Chiers. D'autre part, la vallée d'Anderny-Malavillers se situe au point d'inflexion vers le SW de la limite orientale du bassin versant mosan, si bien que plusieurs auteurs ont envisagé un prolongement amont, vers le SE, des affluents de la Chiers, comme l'Othain et le Loison (fig. 1) (Tricart, 1948 ; Harmand, 1992).

L'interruption de l'écoulement subaérien dans les vallées encaissées dans les calcaires bajociens est de toute évidence liée à la karstification dans ces calcaires. L'analyse morphologique détaillée de la vallée d'Anderny-Malavillers a pour buts de préciser les grandes étapes de l'incision, les liens entre l'incision et la karstification, et l'évolution de la limite de bassin versant dans la région de Briey.

\section{Les plateaux karstifiés}

\section{A. Un karst sous couverture active}

14 Le développement du karst ainsi que l'écoulement subaérien trouvent leur origine dans la fourniture d'eau par cette couverture semi-perméable : par drainance vers le toit du calcaire, et par émergence sur les versants. Tant du point de vue hydrologique que du point de vue morphologique, cette couverture, qualifiée d'active (Caramelle, 1998) a favorisé la formation d'un cryptokarst.

\section{Formes et phénomènes karstiques (fig. 3)}

15 Le karst sous couverture active se traduit par un nombre restreint de formes. Ce sont essentiellement des dépressions fermées, rarement prolongées par un gouffre. Les systèmes souterrains révélés par traçages n'ont été atteints que grâce à des travaux miniers (Joly, 1927 ; Maubeuge, 1956 et 1968) à proximité immédiate du secteur étudié.

De même les relations de ces formes à l'hydrologie et à la morphologie permettent de définir un nombre restreint de phénomènes karstiques. Les mardelles sont des dépressions fermées qui ne s'ouvrent que dans les Marnes de Gravelotte et autres formations marno-argileuses. Elles servent de réceptacle aux précipitations, au ruissellement diffus et peut-être aux suintements des marnes. Evapotranspiration et abaissement des surfaces libres les assèchent le plus souvent en été. Les gouffres s'inscrivent plus profondément dans les assises calcaires (- $44 \mathrm{~m}$ au Gouffre Armand) mais s'ouvrent toujours dans les Marnes de Gravelotte dont ils reçoivent une alimentation pérenne.

17 Les points d'absorption des eaux, à fonctionnement temporaire, sont de deux types : les dolines-pertes et les pertes diffuses. Les dolines-pertes s'ouvrent dans les Marnes de Gravelotte et récupèrent les écoulements organisés générés par les différentes assises marneuses. L'amincissement de la couverture facilite l'absorption totale de ces apports ; le maintien d'une couverture plus épaisse en fait des pertes partielles. Leur fouille a toujours montré l'existence d'un dense réseau de fissures karstifiées ou de véritables conduits dans l'Oolithe de Jaumont (Joly, 1927).

18 Les pertes diffuses, caractéristiques des affleurements calcaires, ne se signalent par aucune morphologie de surface (excepté dans certaines portions de la vallée d'Anderny) mais marquent une forte propension à la migration. Les fouilles entreprises sur la perte du Rouau, située dans la vallée sèche, au Sud immédiat d'Anderny, n’a 
montré sous le «manteau de pierrailles et limons assez épais » qu'« un lacis de fines diaclases » (Maubeuge, 1956).

Enfin quelques dolines sensu stricto s'ouvrent à la surface de l'Oolithe de Jaumont.

20 Au-delà de leurs différences, ces phénomènes procèdent d'une même dynamique génétique, liée à la présence puis au décapage plus ou moins poussé des formations de couverture du calcaire.

\section{Dynamique du karst couvert de la vallée d'Anderny}

21 Les mardelles ne sont que la répercussion en surface de tassements et phénomènes de suffosion corrélatifs de la circulation des eaux dans les Marnes de Gravelotte, et à faible profondeur parfois dans l'Oolithe de Jaumont. Par exemple, dans le secteur de Bettainvillers-Tucquenieux, les galeries drainant le ruissellement souterrain (Mangin, 1974) ont été touchées à $-16 \mathrm{~m}$ au gouffre émissif dit Point $3 \mathrm{~A}$, et à $-8 \mathrm{~m}$ dans l'inversac dit Point S4 (Joly, 1927). Ces mardelles vont ensuite évoluer soit en dolineperte, soit en gouffre.

22 L'évolution en doline-perte semble commandée par l'amincissement de la couverture marneuse qui facilite les liaisons hydrauliques avec l'endokarst. Les suintements permanents des Marnes de Gravelotte accélèrent la karstification des fissures du calcaire qui, à leur tour, permettent l'évacuation plus efficace des matériaux meubles fournis par la couverture. Le calcaire devient apparent en fond de dépression fermée et cette doline peut devenir l'exutoire d'un réseau élémentaire de ruissellement. L'exokarst devient un niveau de base local pour les glacis ou versants qui entourent avant-buttes et buttes-témoins, tandis que l'endokarst évacue alluvions et colluvions. Cette évolution peut être quasi-instantanée comme le signale JOLY à deux reprises : en avril 1925 «le ruisseau longeant les cités de Saint- Pierremont est subitement absorbé par un "trou" »; en décembre 1925 s'ouvre « un trou absorbant " les eaux issues du Point 3A (Joly, 1925-1927).

23 Avec le temps, la distribution des formes karstiques se rapproche de celle que l'on connait dans les karsts de contact lithostratigraphique (Gamez, 1991). Mais dans le cas qui nous intéresse, il s'agit bel et bien d'un cryptokarst généré sous couverture et ne se révélant que postérieurement en surface. En lieu et place du « liseré karstique " propre aux karsts de contact, on trouve ici des phénomènes disposés en champs parfois étendus sur les affleurements de couverture (fig. 3). Un autre argument est fourni par la dynamique des gouffres dont les ouvertures inopinées alimentent le corpus légendaire du Pays-Haut (Trou d'Anderny dit de la Dame Marion) ou sont plus prosaïquement relatées par les géologues. «Un peu plus au NE que la perte des Awis, un gouffre s'est formé en plein Bajocien supérieur marneux, le 24 décembre 1967 ». En 1965, près de Bettainvillers s'ouvrait « un gouffre assez important » dans lequel un «suintement aquifère non négligeable se perdait dans la diaclase coupant le massif calcaire, issu de la base des Marnes de Gravelotte " (Maubeuge, 1968). Ces observations convergentes semblent valider le caractère cryptokarstique de ces phénomènes, ainsi que le caractère récent de la phase de karstification liée au décapage partiel de la couverture qui, trop épaisse, limiterait la drainance vers le calcaire au profit des émergences de versants marneux.

24 À l'opposé, deux arguments peuvent conforter cette hypothèse. Le premier concerne les dolines sensu stricto, hydrologiquement inactives, cantonnées aux zones de plus 
large décapage de la couverture et aux secteurs tectoniquement surélevés : nous les interprétons comme des héritages d'une période de recouvrement plus étendu. Le second s'appuie sur le fait qu'aucun phénomène karstique n'est connu sur les surfaces structurales calcaires exhumées du revers de la côte du Bajocien septentrionale. Pourtant, à plus de $400 \mathrm{~m}$ d'altitude, à $15 \mathrm{~km}$ plus au nord, existent des paléokarsts à remplissages ferrugineux cuirassés (Gamez, 1999, in Faber et al., 1999) datés du Tertiaire. On est donc face à un hiatus karstique de l'ordre de dizaines de millions d'années, dont l'explication tient vraisemblablement aux modalités de dégagement dans le temps de la couverture marneuse.

\section{B. Un karst fortement anthropisé}

Quoiqu'assez discret sous sa couverture marneuse, ce karst pose, avec l'exploitation minière séculaire, des problèmes d'environnement dans cette partie du bassin ferrifère.

En liaison avec l'évolution des pratiques agricoles, on assiste à une utilisation de plus en plus fréquente du karst comme exutoire des systèmes de drainage (perte des Awis, Trou d'Anderny). De même, la pratique consistant à décaper les formations superficielles couronnant le calcaire pour favoriser l'infiltration d'un ruissellement accéléré par la pente et la taille grandissante des parcelles, se répand également, par exemple dans le vallon affluent de la vallée d'Anderny au Sud du Haut de Linière, et le vallon au Sud de Malavillers.

Mais se sont surtout les rapports du karst et des travaux miniers qui préoccupent plus spécialement les populations locales.

À la suite de l'ouverture d'un gouffre ouvert à Mance en 1967, « les habitants voulaient incriminer les travaux miniers alors qu'il n'y en a aucun à la verticale ou aux alentours immédiats » (Maubeuge, 1968). Il en allait de même à l'ouverture brutale de la perte des cités de Saint-Pierremont en 1925 «au-dessus d'une région de la mine qui n'est pas exploitée » (Joly, 1925-1927).

Il est pourtant certain que l'exploitation du minerai aalénien pendant environ un siècle s'est traduite par de fréquentes ruptures des écrans marneux qui isolaient la formation ferrifère des aquifères sus jacents plus ou moins karstifiés. En conséquence les tassements et effondrements d'origine minière sont une réalité, même si les particularités morphologiques et hydrologiques du karst ont été occultées de la mémoire collective avec le temps (Gamez, 1998).

L'étude des résultats de traçages commandés par les exploitants miniers inquiets de voir leurs mines envahies par les eaux (Joly, 1925-1927), révèle une situation complexe (tableau I) : on note un net renversement de tendance entre la première période qui correspond aux premières exploitations dans la région, et la seconde qui se termine par l'apogée de l'extraction minière en Lorraine. Même si les relations hydrologiques exokarst/ galerie de mine ne sont pas une fatalité, on mesure nettement la progression des perturbations hydrogéologiques avec l'extension des travaux miniers. Il conviendra de réévaluer ces rapports après la fermeture récente des mines, l'arrêt des pompages d'exhaure, et la stabilisation de l'ennoyage des vides miniers. 
Tableau 1 : Résultats des traçages dans le bassin versant du Ruisseau de la Vallée

\begin{tabular}{|c|c|c|c|c|}
\hline Période & $\begin{array}{c}\text { Nombre } \\
\text { de traçages }\end{array}$ & $\begin{array}{c}\text { Positifs } \\
\text { dans le karst }\end{array}$ & $\begin{array}{c}\text { Positifs } \\
\text { dans les mines }\end{array}$ & $\begin{array}{c}\text { Négatifs } \\
\text { dans les mines }\end{array}$ \\
\hline $1925-1927$ & 42 & $40^{*}$ & $5^{* *}$ & $69^{* *}$ \\
\hline $1952-1962$ & 25 & $8^{*}$ & $32^{* *}$ & $48^{* *}$ \\
\hline
\end{tabular}

* Résultats; ; En \%.

\section{Conclusion partielle}

31 Les formes et phénomènes karstiques du centre du Pays-Haut appartiennent à un type de karst sous couverture concordante dont la dynamique morphologique est triple.

1) Conservée dans sa totalité, comprenant les marnes du Bajocien supérieur et les faciès marno-calcaires du Bathonien, elle inhibe la karstification, mais en revanche, commande le modelé des versants et favorise l'écoulement superficiel.

2) Lorsqu'elle est résiduelle, la couverture, réduite aux Marnes de Gravelotte devient active et génère un cryptokarst dans des calcaires de Jaumont sous-jacents, puisqu'elle fournit directement l'eau nécessaire à la karstification. Ce cryptokarst, constitué de phénomènes karstiques assez étendus dans l'espace, évolue de mardelles en dolinespertes ou en gouffres, au fur et à mesure du décapage progressif de la couverture marneuse.

3) Éliminée de la surface structurale des calcaires de Jaumont, elle ne se signale plus qu'indirectement grâce aux formes paléokarstiques qui jalonnent dans le temps et dans l'espace les grandes étapes de son recul.

Dans les fonds de vallée principalement, le fonctionnement du karst a été perturbé surtout par l'exploitation du minerai de fer aalénien et ses conséquences : tassements et effondrements, pompages d'exhaure et d'ennoyage des mines après leur fermeture.

Karst sous couverture et perturbations d'origine anthropique coexistent dans le bassin versant de la vallée d'Anderny-Malavillers, où dolines-pertes et pertes diffuses absorbent les eaux superficielles.

\section{III. Évolution morphologique de la vallée d'Anderny- Malavillers}

37 Les processus karstiques fonctionnant actuellement permettent d'expliquer l'assèchement de la vallée d'Anderny-Malavillers, drainée, dans sa section sud-est, seulement une dizaine de jours par an. Ces processus karstiques, confrontés au recul pléistocène de la couverture marneuse du Bajocien supérieur et du Bathonien, permettent également de reconstituer les principales phases de l'inversion du drainage dans la vallée, et de chiffrer l'ampleur du déplacement de la limite de bassin versant Meuse/Rhin pendant le Quaternaire.

\section{A. La morphogenèse actuelle}

Les processus de fonctionnement de la vallée d'Anderny-Malavillers ont été analysés dans la section de vallée du Rouau (fig. 4, 5, 6A). Celle-ci coïncide avec une courbure 
marquée correspondant à une légère gouttière structurale, et avec un fond de vallée étroit ne dépassant pas $10 \mathrm{~m}$ de largeur, bordé de versants raides $(15 \mathrm{~m}$ d'encaissement pour une pente de $12 \%$ environ), modelés en terrassettes d'origine probablement anthropique. En outre, cette section de la vallée au pendage faible, présente un nombre élevé de pertes diffuses. En effet, le fond de vallée, parcouru de plusieurs chenaux, est accidenté de dépressions de dimensions métriques correspondant à des secteurs de pertes diffuses. Les plus caractéristiques d'entre elles ont la forme de petits amphithéâtres de 1 à $5 \mathrm{~m}$ d'arc et de 0,5 à $1 \mathrm{~m}$ de hauteur. Ces derniers correspondent à des micro-chutes spectaculaires au moment des écoulements, comme ce fut le cas le 12 avril 1998. Ces micro-chutes apparaissent donc comme des entailles dans la formation de fond de vallée à granularité sablo-limoneuse (Médiane : 0,3 $\mathrm{mm}$ ) et décalcifiée (teneur en $\mathrm{CaCO} 3:$ 3,75\%). Au pied des micro-chutes existent de petites barres alluviales constituées par une formation à granularité limoneuse dominante (Médiane : 0,076 $\mathrm{mm}$ ), hétérométrique (indice de Trask : 8,3 contre 4,5 pour le remplissage de fond de vallée) et carbonatée (teneur en $\mathrm{CaCO}: 17,5 \%$ ).

Fig. 5 : Carte du fond de la vallée d'Anderny au lieu-dit « le Rouau »

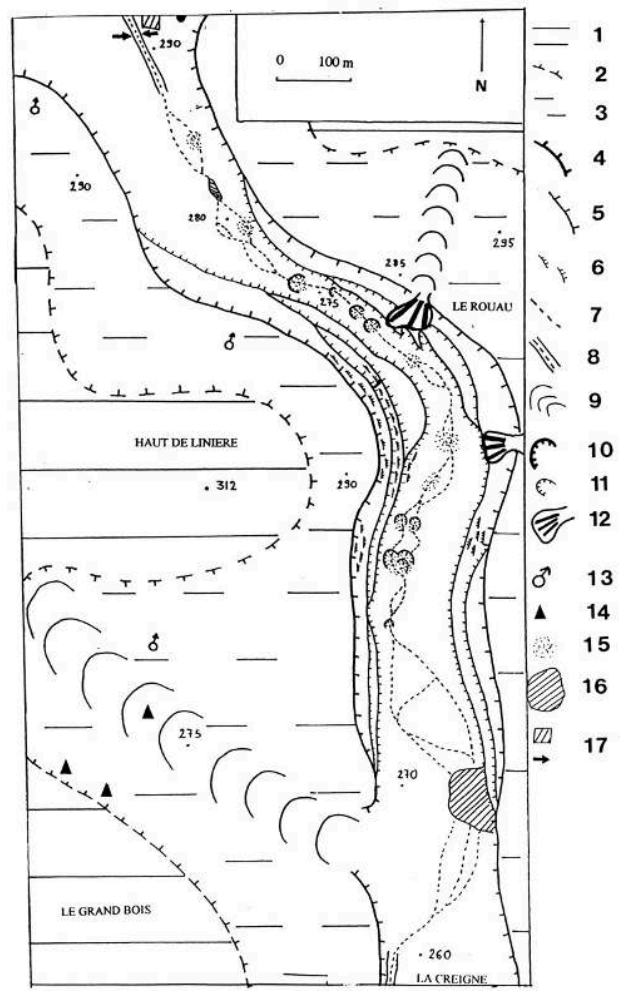

1 : versants modelés dans les Marnes du Jarnisy, 2 : base des versants, 3 : glacis-terrasse dans les Marnes de Gravelotte, 4 : rebord du glacis-terrasse, $5:$ rebord des terrasses dominant le fond de vallée, 6 : rebord des terrassettes, 7 : chenal d'écoulement, 8 : fossé de drainage, 9 : vallon non drainé, 10 : micro-chute en forme d'hémicycle (4-5 m d'arc), 11 : micro-chute en forme d'hémicycle (1-4 m d'arc), 12 : cône alluvial, 13 : émergence, 14 : perte importante, 15 : zones de pertes diffuses, $16:$ mare temporaire, 17 : égouts et effluents issus de la ferme d'Anderny. 
Fig. 6 : Carte structurale du centre du Pays-Haut (A) et cartes de l'extension minimale des formations du substratum (Oolithe de Jaumont, Caillasse à Anabacia, Caillasse à Rhynchonelles et Callovien) aux stades de $340 \mathrm{~m}$ (B) et de $280 \mathrm{~m}$ (C).
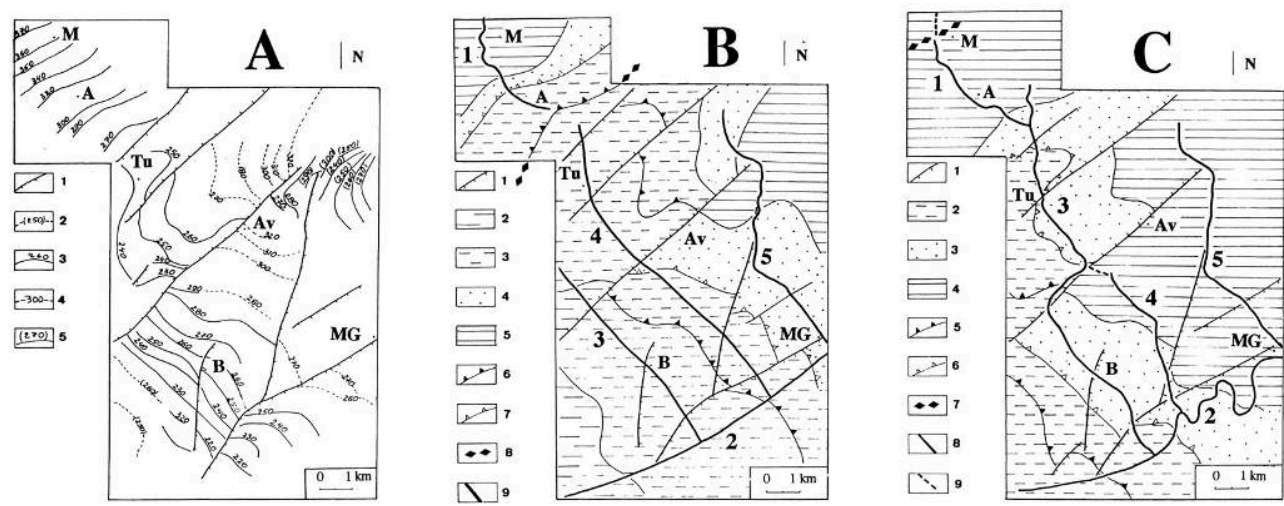

A : 1) principales failles et direction de leur regard, 2) isohypse au toit des Caillasses à Anabacia, 3) isohypse au toit de l'Oolithe de Jaumont, 4) isohypse au toit des calcaires siliceux, 5) isohypse au toit des calcaires à Polypiers (d'après Le Roux, 1983 ; Caramelle, 1998) ;

$B: 1$ ) principales failles et direction de leur regard ; 2 à 5 : extensions minimales des affleurements : 2) des argiles du Callovien, 3) des marno-calcaires du Bathonien, 4) des marno-calcaires du Bajocien supérieur, 5) des calcaires du Bajocien; 6 et 7 : position minimale des microcôtes : 6 ) des Caillasses à Rhynchonelles, 7) des Caillasses à Anabacia ; 8 : limite présumée des bassins versants Meuse/Rhin, 9 : paléoécoulements supposés (9.1 : d'Anderny-Malavillers, 9.2 : Paléo-Orne, 9.3 : Paléo-Woigot, 9.4 : Paléo-Ruisseau de la Vallée, 9.5 : Paléo-Conroy).

$C: 1)$ principales failles et direction de leur regard ; 2 à 5 : extensions minimales des affleurements : 2 ) des marno-calcaires du Bathonien, 3) des marno-calcaires du Bajocien supérieur, 4) des calcaires du Bajocien ; 5 et 6 : position minimale des microcôtes : 5) des Caillasses à Rhynchonelles, 6) des Caillasses à Anabacia ; 7 : limite présumée des bassins versants Meuse/Rhin, 8 : paléoécoulements supposés (8.1: d'Anderny, 8.2: Orne, 8.3 : Ruisseau de la Vallée-Woigot, 8.4 : Ruisseau du Fond de la Noue, 8.5 : Conroy), 9 : vallées sèches ; A : Anderny, Av : Avril, B : Briey, M : Malavillers, MG : MoyeuvreGrande, Tu : Tucquenieux.

L'existence de ces deux types de formations est liée aux processus de fonctionnement de la vallée : la présence d'une nappe alluviale de plus d'un mètre d'épaisseur témoigne d'un colmatage notable de cette section de vallée. Les caractéristiques sédimentologiques de la formation alluviale: prédominance d'éléments fins, mal classés, dans laquelle se trouvent quelques cailloux et blocs de calcaires sub-émoussés, d'une part, ainsi que la présence de petits cônes au débouché de vallons entaillant le versant est de la vallée, d'autre part, privilégient une mise en place sous forme de coulées de particules (Mietton et al., 1998). La décarbonatation de cette nappe alluviale a de toute évidence été favorisée par l'intensification des flux hydriques en fonction du soutirage karstique, d'autant plus efficacement que cette section de vallée est à faible pente.

La formation des barres alluviales est, quant à elle, liée à des processus actuels et à une recharge en éléments fins et en carbonates issus du ruissellement diffus sur les versants modelés dans les Marnes de Gravelotte et du Jarnisy.

La morphogenèse actuelle est indissociable de l'évolution des aquifères des calcaires bajociens. En effet, le dépilage des mines de fer exploitant la couche de l'Aalénien, situées sous ces calcaires, a eu pour conséquence un abaissement de leurs niveaux piézométriques, si bien que les zones d'absorption situées dans les dépressions se trouvant au pied des micro-chutes ne peuvent fonctionner qu'en pertes diffuses. En revanche, l'ennoyage actuel des mines pourra provoquer une remontée des niveaux 
aquifères du Bajocien, en particulier celui des calcaires de Jaumont. Ainsi, les pertes du Rouau pourraient fonctionner alternativement en tant que pertes, pendant la plus grande partie de l'année et en tant qu'exutoires de la nappe de l'Oolithe de Jaumont, notamment lors des plus fortes précipitations de saison froide. Si cette hypothèse se vérifiait, il s'agirait d'inversacs.

En outre, l'existence de pertes, absorbant les eaux usées du village d'Anderny, pose un problème environnemental sérieux, puisque ces dernières contaminent inévitablement la nappe du Bajocien, qui est utilisée pour l'alimentation en eau potable de nombreuses communes de l'Ouest du département de la Moselle.

\section{B. Évolution pléistocène du drainage dans la région d'Anderny}

Les cartes structurales du bassin versant de la vallée d'Anderny (Caramelle, 1998), et de la partie nord-est de la feuille de Briey (Le Roux, 1983) (fig. 6A) permettent de retracer les extensions minimales des formations du substratum à partir de quelques altitudes données, suivant la méthode exposée par Le Roux et Harmand (1998). Comme il s'agit des extensions minimales des formations du substratum pour une région supposée plane, il est nécessaire de tenir compte d'une plus grande extension de la couverture marneuse (Marnes du Jarnisy). De même, il faut prendre en compte l'existence des buttes-témoins des Caillasses à Anabacia qui s'élevaient au-dessus de la surface structurale de l'Oolithe de Jaumont, à l'image de celles qui existent aujourd'hui dans la région d'Anderny.

Deux altitudes ont été choisies : $340 \mathrm{~m}$, altitude du col de Malavillers et $280 \mathrm{~m}$, altitude de la surface structurale de l'Oolithe de Jaumont au-dessus de la section de vallée du «Rouau».

La carte du stade de $340 \mathrm{~m}$ (Fig. 6B) met en évidence une large extension vers l'est des formations essentiellement marneuses du Bathonien, et une avancée des côtes mineures des Caillasses à Anabacia et des Caillasses à Rhynchonelles, notamment dans le fossé tectonique de Tucquenieux. Ce dernier correspondait alors à un point topographiquement haut, et donnait lieu à une divergence de drainage. Les niveaux aquifères du Bathonien (Caillasses à Rhynchonelles et à Anabacia), et du Bajocien supérieur (Marnes du Jarnisy) de la région d'Anderny alimentaient, d'une part, un paléo-écoulement vers le NW, dans la vallée d'Anderny-Malavillers, et d'autre part, des paléo-écoulements vers le SE. Ces derniers, souvent orthoclinaux et orientés du NW au $\mathrm{SE}$, rejoignaient la Paléo-Orne, qui, dans la région de Briey drainait les formations marneuses du Bathonien et du Bajocien supérieur, à l'image de la région aujourd'hui située à l'Ouest de Jarny.

Au stade de $340 \mathrm{~m}$, la vallée d'Anderny-Malavillers était donc drainée par un cours d'eau local, bien alimenté par les aquifères du Bathonien et du Bajocien supérieur. La partie supérieure de la vallée d'Anderny-Malavillers, modelée dans les formations marneuses du Bajocien supérieur, et étanchéifiée par un substrat peu perméable fournissant des particules fines abondantes, devait être drainée en permanence. En revanche, sa partie inférieure, en aval d'Anderny, était encaissée dans les calcaires bajociens. Il est donc probable qu'un soutirage ancien par le karst, en direction de la Crusnes, se soit effectué à cette époque.

Mais surtout, la reconstitution de la limite des couches bajociennes et bathoniennes du stade de $340 \mathrm{~m}$ montre que la limite des bassins versants de la Meuse et du Rhin se 
situait à 2 ou $3 \mathrm{~km}$ seulement au SE d'Anderny. Par conséquent, une éventuelle extension de la vallée d'Anderny-Malavillers vers le SE, aux dépens du bassin versant de l'Orne, ne peut être reconstituée.

En outre, la reconstitution du stade $340 \mathrm{~m}$ implique, qu'au NW du Fond de Maufontaine, la vallée d'Anderny-Malavillers était encaissée dans les calcaires bajociens. Aussi, l'existence d'une vallée encaissée dans les calcaires au SE de l'actuelle limite des bassins versants de la Meuse et du Rhin a préparé le renversement de drainage au profit du Ruisseau de la Vallée.

L'absence de restes de paléo-nappes alluviales correspondant à de hautes terrasses dans les vallées de l'Orne et de la Moselle ne permet pas de dater avec précision les réorganisations de drainage de la région de Briey. Toutefois, l'âge présumé pliocène terminal des alluvions situées à $340 \mathrm{~m}$ dans la vallée de la Moselle, dans la région de Trèves, indique que l'abandon du Col de Malavillers, situé à $340 \mathrm{~m}$ à plusieurs dizaines de kilomètres à l'amont, est probablement d'âge pléistocène. Cette hypothèse tient compte de la déformation éventuelle des hautes terrasses de la vallée de la Moselle entraînées dans le soulèvement du Massif Schisteux Rhénan (Negendank, 1983). De même, les corrélations effectuées avec les niveaux de terrasses du bassin de la Meuse semblent également confirmer l'âge quaternaire de la réorganisation de drainage de la vallée d'Anderny. En effet, les niveaux de terrasses présumés pliocènes les plus récents se situent vers $285 \mathrm{~m}$ au Sud de la confluence Meuse-Chiers et vers $340 \mathrm{~m}$ dans la vallée de la Chiers, au NE de Longuyon (Pissart et al., 1997 ; Farina, 1999). Ainsi, la paléo-vallée d'Anderny-Malavillers fonctionnait encore durant le Pléistocène inférieur.

La carte du stade de $280 \mathrm{~m}$ se situe dans le temps à une période relativement peu éloignée de l'actuel, puisque la vallée d'Anderny, dans la section du Rouau, n'a été incisée que d'une dizaine de mètres depuis le stade $280 \mathrm{~m}$ (fig. 6C).

51 Dans le bassin versant de l'Orne, l'encaissement des cours d'eau, en réponse à l'incision de la «Paléo-Meurthe » dans la dépression orthoclinale de la côte de Moselle, a pour effet d'entraîner le recul vers le SW des côtes mineures du Bathonien et des contacts entre marnes et calcaires. Ainsi, l'Orne et son affluent le Conroy sont encaissés dans les calcaires bajociens dès avant le stade de $280 \mathrm{~m}$, à l'Est du méridien d'Avril. En outre, la mise à l'affleurement des calcaires bajociens au SW d'Avril a entraîné une réorganisation de drainage le long de la faille d'Avril, aux dépens de la partie inférieure du Ruisseau de la Vallée encaissée dans les calcaires siliceux d'Orne, au profit du Woigot. Dans la région d'Anderny, le recul de la couverture marneuse s'est poursuivi, à tel point que les Marnes de Gravelotte affleuraient, dès le stade de $280 \mathrm{~m}$, dans le fond de la vallée d'Anderny, au lieu dit les Rouau, et dans celui de la vallée de Brabant, au Nord des cités ouvrières.

52 La carte du stade de $280 \mathrm{~m}$, confrontée à la carte géologique actuelle et aux conclusions de la deuxième partie, permet ainsi de reconstituer les étapes de l'inversion de drainage dans la paléovallée d'Anderny-Malavillers :

53 A) Dans un premier temps, l'abaissement des interfluves lié à l'incision dans la vallée de l'Orne et la réduction d'épaisseur de la couverture marno-calcaire rédui- sent considérablement l'impluvium dans la paléo-vallée d'Anderny-Malavillers et entraînent l'assèchement progressif de la partie supérieure de la paléo- vallée.

54 B) Dans un deuxième temps, l'inversion de drainage est liée à l'érosion régressive dans le Ruisseau de la Vallée et le Ruisseau des Cités de Brabant. Dès que les Marnes de 
Gravelotte sont à l'affleurement dans le fond de la vallée, la karstification est initiée sous couverture active (stade de $280 \mathrm{~m}$ ). Cette karstification s'effectue probablement en relation avec un système de fractures de direction méridienne. En effet, actuellement un écoulement souterrain de direction subméridienne, mis en évidence par des traçages, suit l'axe de la vallée du Ruisseau des Cités de Brabant (fig. 3).

c) Dans un troisième temps, l'inversion de drainage proprement dite dans la vallée d'Anderny a été facilitée par plusieurs facteurs : a) existence d'un pendage incliné vers le SE et présence d'une petite gouttière synclinale qui a canalisé les écoulements, b) rôle de la surface structurale des Marnes de Gravelotte qui a canalisé les écoulements subaériens, c) rôle de ces mêmes Marnes de Gravelotte en tant que couverture active, d) enfin, permanence de l'écoulement dans le Ruisseau de la Vallée bien alimenté par les aquifères de la couverture marno-calcaire largement conservée.

D) Dans un quatrième temps, l'amincissement de la couverture des Marnes de Gravelotte lié à l'érosion régressive dans le bassin de l'Orne accélère la dynamique karstique : les pertes localisées dans les calcaires apparaissant dans le fond de la vallée font transiter par l'endokarst les matériaux fins fournis par les versants, tandis qu'en période de hautes eaux, ces mêmes matériaux fins sont évacués le long de la vallée.

E) Dans un cinquième temps, en aval d'Anderny, la vallée d'Anderny est surcreusée d'une dizaine de mètres en contrebas du toit de l'Oolithe de Jaumont. L'incision dans les calcaires de Jaumont et le recul de la couverture marneuse sont liés, quant à eux, au drainage partiel de la vallée par de petits ruisseaux issus des secteurs où cette couverture est encore épaisse.

\section{Rôle présumé des alternances climatiques du Quaternaire}

L'évolution morphologique de la vallée d'Anderny-Malavillers doit être replacée dans le cadre des alternances de périodes froides et tempérées du Quaternaire. L'incision dans les calcaires du Bajocien inférieur et moyen s'est, de toute évidence, effectuée lors des périodes froides à pergélisol, comme en témoigne la morphologie de la vallée d'Anderny-Malavillers, notamment au NW du village d'Anderny, de même qu'un grand nombre de vallées du Pays-Haut : cette morphologie de vallées à versants raides et à fond plat, typique des paléo-environnements périglaciaires des plateaux calcaires (Tricart, 1981), est due notamment à l'action du ruissellement sur sol gelé et d'un sapement du pied du versant.

De même, l'évolution des versants argilo-marneux a probablement été exacerbée sous les paléo-environnements périglaciaires, dans la mesure où les formations du Bajocien supérieur et du Bathonien ont été nécessairement affectées par la gélifluxion.

La karstification a, quant à elle, été davantage favorisée pendant les périodes tempérées. Par exemple la création et et la conservation des mardelles ne peut se concevoir en périodes de processus de versant exacerbés. Mais la karstogénèse est également admise lors des périodes froides. En effet, on peut supposer que, lors des périodes à pergélisol peu profond, les Marnes de Gravelotte ont partiellement protégé la masse calcaire des ondes de froid, et pu générer des écoulements au moins saisonniers vers le cryptokarst incipient. 


\section{Conclusion partielle}

61 Ainsi, la réorganisation du drainage de la région de Briey s'effectue sous commande structurale et plus précisément correspondant au passage d'un relief inverse à un relief conforme dans le fossé de Tucquenieux. Au début du Pléistocène, la limite des bassins versants de la Meuse et du Rhin coïncidait avec ce fossé dans lequel était conservé un système de butte-témoins de la côte mineure des Caillasses à Rhynchonelles. L'inversion de drainage de la vallée d'Anderny correspond ainsi à la mise en relief de l'ondulation anticlinale d'Audun-le-Roman par décapage de la couverture marnocalcaire vers le SE. Mais cette réorganisation de drainage est locale, puisque la limite des bassins versants de la Meuse et du Rhin n'a guère reculé depuis cette époque.

De même, l'évolution du drainage est liée à l'amincissement de la couverture argilomarneuse du Bajocien supérieur et du Bathonien et à une karstification des calcaires de Jaumont par la mise à l'affleurement des Marnes de Gravelotte.

63 L'érosion régressive liée au niveau de base de l'Orne et l'abondance des écoulements superficiels bien alimentés par les aquifères de la couverture marno-calcaire largement conservée, sont, quant à elles, à l'origine de l'inversion du drainage.

\section{Conclusion générale}

À travers l'étude comparative des dynamiques fluviale et karstique de la vallée d'Anderny-Malavillers, il est possible de proposer quelques jalons pour l'évolution morphologique du Pays-Haut et de la Lorraine septentrionale. Cette évolution est marquée par la dualité de l'impact des binômes de résistance lithologique sur le relief et l'hydrologie. Elle induit une rythmicité morphogénétique qui se traduit par le passage d'un relief conforme à un relief inverse et vice versa. Ceux-ci à leur tour privilégient l'action d'une morphogenèse aboutissant à un modelé commandé tantôt par l'action du réseau hydrographique, tantôt par l'action prépondérante du karst.

Soumis à la règle de l'immunité karstique, le plateau de revers de la côte du Bajocien offre une variété de formes qui ont enregistré les grandes étapes de son façonnement liées aux rythmes du recul de sa couverture marneuse. L'ablation totale de cette dernière n'est réalisée qu'en amont-pendage, à proximité immédiate du front de la côte. Deux générations de paléo-karsts tertiaires y coexistent. La première est caractérisée par un remplissage de type ferricrète en place (Borne de Fer ; Fig. 1). Elle est interprétée comme un héritage paléo à médio-tertiaire (Gamez, in Faber et al., 1999), aujourd'hui placé, à l'échelle régionale, en inversion de relief, puisque situé dans l'ondulation synclinale du Luxembourg. La seconde est caractérisée par le piégeage de cette même formation remaniée, qui fossilise une cinquantaine de mètres en contrebas une deuxième génération de formes karstiques, comme à Saint-Pancré. Jalonnant la surface dite de $400 \mathrm{~m}$, présumée oligo-miocène, cette karstification serait d'âge néogène.

Une troisième génération de formes karstiques se retrouve en lisière de la vallée d'Anderny-Malavillers. Les dolines sensu stricto, sans activité hydrologique, correspondent à des héritages crypto-karstiques. Ils marquent un recul significatif des marnes du Bathonien et du Bajocien supérieur qui ne laissait plus à la surface du calcaire que la couverture active des Marnes de Gravelotte. La karstification de 
l'Oolithe de Jaumont sous-jacente pouvait débuter. Selon toute vraisemblance cette phase pourrait être placée à la charnière Tertiaire-Quaternaire, ou dans la première moitié de ce dernier. Jusqu'alors la vallée d'Anderny-Malavillers formait une seule et même unité hydrographique tributaire de la Meuse, s'écoulant sur la couverture marneuse.

L'étape suivante est à placer résolument dans le Quaternaire. Elle correspond à l'individualisation de la vallée d'Anderny sensu lato à partir du « col » de Malavillers et à une quatrième génération de karstification. Cette dernière a engendré les formes et phénomènes karstiques actuellement observables.

Enfin, au cours du XXe siècle, le karst couvert est perturbé par les interventions de l'homme, qui sont ainsi à l'origine d'une cinquième phase de karstification. Ces perturbations anthropiques, principalement d'origine minière, risquent à court terme de remettre en cause la stabilité relative de la limite de bassins versants Meuse-Moselle au profit de la seconde.

La réorganisation du drainage de la région de Briey apparaît, quant à elle, similaire à celle de la région du site de capture de la Haute Moselle (Le Roux et Harmand, 1998). Dans les deux cas, les captures s'effectuent au profit des affluents surimposés les plus importants de la "Paléo-Meurthe ", puis de la Moselle. Des cours d'eau alimentés dans leur partie supérieure par la nappe aquifère des calcaires de la côte de Meuse, et par le drainage des Argiles de la Woëvre, comme l'Orne, réorganisent le drainage à leur profit au cours de leur encaissement dans les calcaires bajociens. Nous pensons que la dynamique fluviale de l'Orne est due au recoupement de la nappe aquifère du Bajocien, à l'image de ce qui a été proposé pour l'Esch (Sary et al., 1992).

Toutefois, une différence essentielle oppose la Lorraine septentrionale et la Lorraine méridionale. Au Quaternaire moyen, la Lorraine du Sud a subi, avec la capture de la Haute Moselle, une importante modification du tracé de la limite du bassin versant Meuse-Rhin. En Lorraine du Nord, cette limite semble très ancienne et n'a pas été sensiblement modifiée par des captures durant le Pléistocène.

\section{BIBLIOGRAPHIE}

Bresson G. (1960). - Coupe de la carrière de Jaumont à Saint-Privat-la-Montagne. Archives D.D.A. du département de la Meuse, 1 fig.

Caramelle F. (1998). - Contribution à l'étude du karst du Pays-Haut : le bassin versant de la vallée d'Anderny. Mémoire de Maîtrise de Géographie Physique. Université de Nancy 2, 104 p, 1 carte h. t.

Faber A., Gamez P., Hanzo M., Mirambet F., Amoferlor et Musée du Fer de Jarville (1999). - Le Fer. In Bull. Inf. Géol. Bass. Paris, Vol. 36, n 1, 27-33.

Farina S. (1999). - Contribution à l'étude du bassin moyen de la Chiers. Maîtrise de Géographie physique. Université de Nancy 2.64 p. 
Gamez P. (1991). - Hydrologie et karstologie du bassin du Loison (Woëvre septentrionaleLorraine). Mosella, Tome XXI-parution 1995, 453 p.

Gamez P. (1998). - Quelques problèmes d'aménagement et d'environnement dans les zones karstiques lorraines. In : Arbeiten aus dem Geographischen Institut des Universität des Saarlandes. Symposium « Problèmes de l'environnement en Saar-Lor-Lux », pp. 41-51.

Harmand D. (1992). - Histoire de la vallée de la Meuse lorraine. Presses Universitaires de Nancy (Coll. «Etudes géographiques »), 146 p. : cartes, graph., tabl.

Joly H. (1925-1927). - Étude hydrogéologique de Saint-Pierremont, Tucquenieux, Anderny, Trieux. Notes. Archives Usinor 142/420, fond Musée des mines de Neufchef.

Joly H. (1927). - Les relations des ruisseaux souterrains de la région de Tucquenieux avec les venues d'eaux souterraines. Rev. Ind. Min., 154, pp. 199-218.

Le Roux J. (1980). - La tectonique de l'auréole orientale du Bassin de Paris. Ses relations avec la sédimentation. Bull. soc. géol. France, t. XXII, n 4, pp. 655-667.

Le Roux J. (1983). - Notice explicative de la feuille de Briey de la carte géologique de France à 1/50000. B.R.G.M. Paris.

Le Roux J., Harmand D. (1998). - Contrôle morphostructural de l'histoire d'un réseau hydrographique : le site de la capture de la Moselle. Geodinamica acta, vol. 11, 4, 149-162, 10 fig.

Mangin A. (1974). - Contribution à l'étude hydrodynamique des aquifères karstiques. Ann. Spél., $29,3-4$ et $30,1,258 \mathrm{p}$.

Maubeuge P.L. (1956). - Hydrologie du Bassin Ferrifère Lorrain (première note). Extrait du bulletin technique de la Chambre Syndicale des mines de Fer de France, $\mathrm{n}^{\circ} 42$, pp. 1-21.

Maubeuge P.L. (1968). - Hydrologie du Bassin Ferrifère Lorrain (seconde note). Extrait du bulletin technique de la Chambre Syndicale des mines de Fer de France, ${ }^{\circ}$ 90, pp. 7-25.

Mietton M., Ballais J.-L., Marre (1998). — L'érosion hydrique mécanique et les mouvements de terrain sur les versants et les bassins versants. In : L'érosion entre nature et société, coor. VEYRET Y., SEDES, Dossier des Images Economiques du Monde, 57-107.

Negendank J. (1983). - Sammlung Geologischer Führer. Band 60. Trier und Umgebung, 2 Auflage, $195 \mathrm{p}$.

Pissart A., Harmand D., Krook L. (1997). — L'évolution de la Meuse de Toul à Maastricht depuis le Miocène : corrélations chronologiques et traces des captures de la Meuse lorraine d'après les minéraux denses. Géographie Physique et Quaternaire, vol. 51, n 3, pp 267-284, 10 fig., 1 tabl.

Sary M., Corbonnois J., Gamez P., Gille E. (1992). - Profils hydrologiques de l'Esch in Livret-guide des excursions des Journées hydrologiques de Metz-Arlon. Hydrologie des milieux calcaires en étiage. Centre d'Etudes Géographiques de l'Université de Metz, 2 p.

Steiner P. (1980). - Lithostratigraphie et fracturation du Dogger lorrain. Thèse 3e cycle, Univ. Nancy I, $203 \mathrm{p}$.

Tricart J. (1948). - La partie orientale du Bassin de Paris. Étude morphologique. Thèse Paris (lettres) et S.E.D.E.S. éd. Paris, t. 1 : La genèse du bassin, p. 1-210, fig. 1-30, photogr. 1-8, 1 carte h.t. t. II : l'évolution morphologique au Quaternaire, p. 211-274, fig. 31-90, photogr. 9-24, 1 pl.

Tricart J. (1981). - Précis de géomorphologie. CDU-SEDES. t. 3, 313 p. 


\section{RÉSUMÉS}

Sur le plateau de revers du Pays-Haut, dans l'axe du synclinal de Luxembourg, confondu ici avec le synclinal faillé d'Ottange-Tucquenieux, les calcaires du Bajocien moyen et supérieur sont recouverts par les marnes du Bajocien supérieur et du Bathonien. Le secteur d'Anderny se caractérise par l'existence d'une vallée sèche héritée du Pliocène, traversée par la ligne de partage des eaux de la Meuse et du Rhin et la présence de nombreux phénomènes karstiques. Fin Tertiaire, le recul de la couverture marneuse et des côtes mineures de Caillasses à Anabacia et à Rhynchonelles dans le synclinal faillé de Tucquenieux, passant d'un relief inverse à un relief conforme, a entraîné l'inversion du drainage dans la "vallée d'Anderny ». Par contre, la limite de bassin versant n'a guère été modifiée au cours du Pléistocène. La présence de nombreux intercalaires carbonatés (Marnes de Gravelotte, Caillasse à Anabacia, Caillasse à Rhynchonelles) dans une couverture marneuse épaisse a favorisé par drainance la karstification des calcaires sous-jacents (Oolithe de Jaumont). L'amincissement progressif de la couverture marneuse entraîne l'arrêt des écoulements dans les vallons inscrits dans les calcaires et la répercussion en surface des formes endo-karstiques. Les perturbations anthropiques, principalement d'origine minière, entraîneront à terme une modification de l'évolution morphologique de ce milieu.

On the dipslope of the Bajocian escarpment of the Pays-Haut, in the Luxemburg synclinal axis, which corresponds to the faulted syncline of Ottange-Tucquenieux, the Middle and Upper Bajocian limestones are concealed by Upper Bajocian and Bathonian marls. The district of Anderny is characterised by the existence of a Pliocene dried valley crossed by the drainage line of the Meuse and the Rhine, and the presence of a number of karstic phenomena. At the end of the Tertiary the retreat of the marl cover and of the micro-escarpments of the Caillasse à Anabacia and the Caillasse à Rhynchonelles in the faulted syncline of Tucquenieux, changing the relief from inverted to conformable, removed the original northwestern drainage through the Meuse River, to a new southeastern drainage through the Orne and Moselle rivers. On the other hand, the watershed of the basin was hardly changed during the Pleistocene. The presence of a number of carbonate intercalations (Marnes de Gravelotte, Caillasse à Anabacia, Caillasse à Rhynchonelles) in a thick marl cover favoured the karstification of the underlying limestones (Jaumont Oolite). The progressive thinning of the Marl cover led to the arrest of flow in the small limestone valleys and the emergence at the surface of endo-karstic forms. Antropic disturbances, principally by mining, will change the morphologic evolution of this environment.

Auf des Landterrasse des Pays-Haut, in der Synklinalachse von Luxemburg (Richtung Trierer Bucht), die sich hier mit der Bruchfalte von Ottange-Tucquenieux verbindet, werden die Kalksteine des Ober- und Mittelbajocien von den Mergeln des Oberbajocien und der Bathonien überlagert. In der Umgebung von Anderny befindet sich in einer Talung die Wasserscheide zwischen Maas und Rhein mit vielen Karstphänomenen. Das Zurückweichen der Mergeldecke und der Mikrostufen in den Schichten von Caillasse à Anabacia und Rhynchonelles in der Bruchfalte von Tucquenieux und im Übergang von der Reliefumkehr in eine struckturbedingte Form hatte die Entwässerungsumkehr im Anderny-Tal zur Folge. Hingegen ist die Grenze des Wassereinzugsgebiets während des Pleistozäns fast nicht verändert worden. Die Existenz zahlreicher Karbonatkalksteine (Marnes von Gravelotte, Caillasse à Anabacia, Caillasse à Rhynchonelles) in einer dichten Mergeldecke hat durch Sickerwasserzufuhr die Karstbildung der unterlagernden Kalksteine (Oolithe von Jaumont) gefördert. Die fortschreitende Ausdünnung der Mergeldecke hat die Unterbrechung des Abflüsses in den Trockentälern im Kalkstein mit Auswirkung der unterirdischen Karstformen auf die Oberfläche zur Folge. Die meist durch den Bergbau entstandenen anthropogenen Störungen werden wahrscheinlich auch die morphologische Entwicklung beeinflussen. 
INDEX

Schlüsselwörter : Karst, Lothringen, Mikrostufe, Oolithe von Jaumont, Pays-Haut, Reliefumkehr, Strukturform, Trier Bucht, Wasserscheide

Keywords : drainage basin, inverted relief, Jaumont Oolite, karst, Lorraine, Luxemburg synclinal axis, micro-escarpment, Pays-Haut, structural relief

Mots-clés : côte mineure, karst, limite de bassin versant, Lorraine, Oolithe de Jaumont, PaysHaut, relief conforme, relief inverse, synclinal de Luxembourg

\section{AUTEURS}

\section{PATRICE GAMEZ}

Centre d'Études Géographiques de l'Université de Metz - 57045 Metz Cedex 01

\section{DOMINIQUE HARMAND}

Laboratoire de Géographie - Université de Nancy 2, BP 33-97, 54015 Nancy Cedex

\section{FLORENCE CARAMELLE}

Centre d'Études Géographiques de l'Université de Metz - 57045 Metz Cedex 01 / Laboratoire de Géographie - Université de Nancy 2, BP 33-97, 54015 Nancy Cedex 